\title{
Introducción a 'Construyendo la investigación sociocultural en educación matemática'
}

Núria Planas, Universitat Autònoma de Barcelona (España)

\section{El concepto sociocultural en nuestra área de investigación}

Hace tiempo, un doctorando me preguntó qué sentido tenía la investigación sociocultural en educación matemática de la que yo le estaba hablando si investigar consistía en simplificar. Me sorprendió la pregunta y mi respuesta espontánea fue que la investigación sociocultural en educación matemática intenta no simplificar en exceso, no sobre-simplificar. Fue una respuesta trampa ya que el término 'sociocultural' es en sí mismo una simplificación de la relación entre cultura y sociedad. Aquel estudiante finalizaría un trabajo de tesis excelente basado en la detección de comportamientos de alumnos resolviendo tareas matemáticas en clase. Decidiría que los comportamientos se podían documentar con relativa facilidad, mientras que las culturas de participación matemática y las formas de interacción entre alumnos y con el profesor eran difíciles de interpretar con garantías de objetividad. Ciertamente, los comportamientos son visibles y la influencia en ellos de la cultura y de la estructura social no siempre lo es.

El actual monográfico está dedicado a la construcción del dominio de investigación sociocultural en educación matemática y a los desafíos abiertos para comunicar con claridad su sentido. Se plantea una mirada al dominio mediante ocho estudios de autores en Costa Rica, Estados Unidos, Nueva Zelanda, Malta, Líbano, México y España. Es una colección incompleta y esquemática, mediada por la trayectoria de la editora de la revista (ver e.g. Planas, 2010), mi red de colaboraciones como investigadora y mis conversaciones académicas con compañeros y editores asociados. Ahora bien, dados los objetivos que se persiguen con el monográfico, es una colección suficiente. Se pretende ilustrar la diversidad de aproximaciones que conviven dentro del dominio, a la vez que ofrecer un espacio de discusión sobre el concepto sociocultural. Los artículos en su conjunto indican que el concepto sociocultural es compatible con y se está fabricando en la investigación en educación matemática dentro de una variedad de aproximaciones teóricas, relativamente aisladas algunas respecto a otras o en escaso diálogo. Más en general, el monográfico busca contribuir a fortalecer la comprensión y la reflexión sobre aspectos singulares de la investigación sociocultural en educación matemática.

El concepto de que el aprendizaje y la enseñanza de matemáticas son procesos principalmente sociales está en el mapa de la investigación en educación matemática desde hace décadas y ha ido ganando bastante presencia en los últimos años. Lerman (2000) escribió sobre el "social turn" para referirse a las reacciones a la investigación positivista y racionalista en el área, a menudo sustentada en la epistemología piagetiana. A día de hoy, estas reacciones se siguen formulando como respuesta a diagnósticos de una crisis de representación de los problemas de la educación matemática priorizados en nuestra comunidad científica. No pocos autores en el área que describen su investigación reciente como sociocultural, algunos de los cuales se autoenmarcan en la epistemología de la educación matemática crítica (Skovsmose, 1994), han sido formados y tienen recorrido en la tradición positivista, de modo que el giro social se aprecia tanto a nivel colectivo como individual. Incluso fundadores de la investigación cualitativa en 
ciencias sociales como el sociólogo norteamericano Barney Galland Glaser, a menudo conocido por la teoría fundamentada, iniciaron sus aportes con aspiraciones de objetividad y de generalización. Hasta Glaser (2001), el lema “all is data" no se aplicaría a la subjetividad de los participantes en un estudio incluidos los investigadores.

El giro sociocultural en la investigación en educación matemática deconstruye la subjetividad del investigador y sitúa su biografía e individualidad en interacción indivisible con los contextos y participantes que estudia. Esta subjetividad no es un sesgo ni una intrusión, sino más bien el instrumento que hace posible recoger e interpretar datos a partir de los cuales fabricar y comunicar resultados. Influye en el análisis que, por ejemplo, la investigadora que estudia las mujeres zapotecas que leen, escriben y calculan para participar en la economía local y familiar sea maestra de los hijos de estas mujeres; que la investigadora que estudia la entrada y distribución de culturas y formas de actividad colectivistas en clases de matemáticas neozelandesas sea de origen Pāsifika; o que el investigador que analiza patrones en la lengua multilingüe de enseñanza de contenidos de álgebra en escuelas del Líbano sea hablante de las lenguas de los alumnos y no requiera traductores ni facilitadores. La comprensión de cómo la subjetividad del investigador debe hacerse explícita y por qué en el curso de un estudio ha generado y sigue generando aproximaciones distintas dentro del dominio. En cualquier caso, son aproximaciones que no manejan la subjetividad humana como amenaza a la credibilidad científica, ni como falta de objetividad o problema a tratar. La subjetividad humana (que incluye nuestras creencias, con sus contradicciones y convergencias, en el sentido explicado en un artículo de este monográfico) es aquí un mediador más entre los fenómenos que deseamos comprender y el conocimiento que generamos, y esta subjetividad está a su vez mediada por la participación en culturas y estructuras sociales.

\section{2. ¿Qué estamos simplificando/abreviando tras 'sociocultural'?}

Al unir las cualidades de lo cultural y de lo social en la abreviación 'sociocultural' para delimitar la investigación sociocultural en educación matemática, se señala sobre todo la inseparabilidad de la cultura y la estructura social. Cultura y estructura social se co-constituyen, al igual que individuo y sociedad. Este es el punto de partida desde la psicología con Vygotsky (1978) -a pesar de que Vygotsky apenas usó el término sociocultural en su obra- y desde la filosofía con Merleau-Ponty (1964), entre otras disciplinas y autores. Ni el que aprende ni el que enseña matemáticas, ni sus mentes, son entidades ahistóricas flotando en el aire, libres de cultura y estructuras sociales; lo cual sirve también para quien investiga el que aprende y el que enseña matemáticas.

Desde una perspectiva amplia que auna diversas aproximaciones socioculturales, la cultura es un conjunto histórico de estándares situados que se aprenden y se necesitan saber para reconocer las expectativas de los otros y las formas de relacionarse apropiadas (Goodenough, 1971). Así, podemos hablar de culturas veganas, culturas agrícolas, culturas en el lugar de trabajo, culturas del aula de matemáticas, culturas matemáticas, culturas de enseñanza del álgebra, culturas de evaluación del rendimiento escolar... En la investigación sociocultural en educación matemática, es de esperar sin embargo que el concepto de cultura se concrete a la luz de conceptos específicos de la educación matemática tales como los de matemáticas, aprendizaje, enseñanza, instrucción, currículo... De ahí que el significado de cultura sea genérico y no definitivo en el dominio hasta que se explican las teorías en las cuales se piensan otros conceptos más específicos. Lo mismo ocurre con el significado de estructura social y con el de la relación entre cultura y sociedad. Dentro del dominio de la investigación sociocultural 
en educación matemática, estos significados devienen concretos cuando se sitúan en el marco de teorías que significan conceptos específicos de la educación matemática.

La investigación sociocultural en educación matemática que, por ejemplo, conceptualiza el aprendizaje como objetivación cultural del saber (Radford, 2002) estrecha una noción de cultura del aula de matemáticas a modo de configuración de objetos históricamente producidos que sugieren a alumnos y profesores formas de intuir fenómenos de la realidad. Este complejo de objetos configura la cultura del aula como el complejo de células individuales configura el cuerpo del ser vivo llevando consigo una memoria histórica que es genética, si se me permite una metáfora en el reino de la biología. En el artículo del monográfico sobre la lengua para enseñar la relación entre área y volumen, la noción implícita de cultura no se refiere a la producción histórica del aula de matemáticas y de sus procesos de actualización de la memoria colectiva; se toman las culturas pedagógicas con futuras maestras y las culturas curriculares de la educación matemática en las aulas universitaria de formación del profesorado y de la escuela de primaria, y los estándares se analizan desde la perspectiva de su construcción sociolingüística. También es distinta la noción de cultura subyacente a la teoría antropológica de lo didáctico (Chevallard, 1990) y a teorías como la etnometodología y los fondos de conocimiento, que guían otros estudios del monográfico. Mirados los artículos en conjunto, se ve en definitiva que el término sociocultural que utilizamos es otra simplificación para representar una familia de términos que abarca lo históricocultural, lo sociohistórico, lo sociolingüístico, lo antropológicocultural...

Para ambos conceptos, cultura y estructura social, en los distintos artículos se llega a significados dentro de teorías pensadas para investigar fenómenos particulares de la educación matemática. A partir de ahí, se abre un continuo de interpretaciones según cómo comprendamos otros conceptos de la educación matemática, cómo concibamos la relación básica entre cultura y sociedad, y dónde esté nuestro énfasis. Por un lado, el énfasis en lo social destaca que se participa en culturas de práctica matemática por vivir en sociedad y ocupando un lugar en un entramado de estructuras sociales. Por otro lado, el énfasis en lo cultural destaca que dicha participación se organiza con base en reglas que la cultura dicta y que pueden ser excepcionalmente cambiadas. No son énfasis que compitan entre ellos, sino complementarios, como las caras de una moneda si se me permite otra metáfora. Con cualesquiera de los énfasis, se concluye sobre la mayor posibilidad de que se acepten sin cuestionar una matemática escolar -con sus reglas- y un proyecto de educación matemática -con sus reglas-. Pero la matemática escolar y el proyecto de educación matemática que no se cuestionan están lejos de ser fenómenos naturales como el clima o el ciclo del agua; son productos históricos, estandarizados y por ende artificiales, creados en la relación entre cultura y sociedad. Hay más tipos de estructuras aditivas que se podrían trabajar en la escolarización temprana, más tipos de geometrías lineales que se podrían incluir en los currículos y que podrían tener en cuenta que las carreteras rectas no son comunes en todas las partes del mundo. La investigación sociocultural en educación matemática cuestiona las razones por las cuales hay culturas cuyas reglas se consideran racionales, lógicas y universales, tal como se nos recuerda en el artículo con datos de Costa Rica y sus zonas montañosas sin apenas carreteras rectas, o en el artículo con datos de Estados Unidos sobre lo costoso de reemplazar creencias acerca de currículos compensatorios para alumnos en procesos de aprendizaje del inglés.

En el artículo donde se discuten normas y prácticas matemáticas democráticas de aula, queda de manifiesto que la adaptación y adecuación a esas normas y prácticas es un criterio de identificación del alumno 'experto', aquel que conoce bien y acepta las reglas de la cultura del aula y de la matemática escolar. Este o cualquier otro alumno 


\section{Introducción}

tiene un papel activo a la vez que complicado en su aprendizaje matemático. Recrea normas y prácticas y, cuando esta recreación no es adecuada, el profesor tiene la responsabilidad de hacerlo notar sin que esto implique paradójicamente una explicación detallada de la norma o práctica de referencia. Son interesantes las conversaciones iniciadas por alumnos 'no expertos' cuando preguntan “¿por qué?” -¿por qué no está bien mi manera de proceder? ¿por qué debería proceder de otro manera? ¿por qué tengo que usar álgebra si puedo resolver el problema con números? ¿por qué tengo que aplicar el teorema de Pitágoras si puedo llegar a saber el área del cuadrado que se forma? Las respuestas serán lógicas o racionales dentro del sistema de reglas de las culturas que se consideren. Es una situación similar al estudiante de la autoescuela cuando pregunta por qué tiene que abonar el peaje en una autopista. Hay reglas y consecuencias. De distinta índole es la situación del estudiante que pregunta por qué debe parar el coche en un 'stop'; aquí la respuesta tiene más que ver con evitar un accidente y preservar la vida, no tanto con la adecuación a reglas. Si se quiere que el estudiante de matemáticas practique y entienda el teorema de Pitágoras, tal vez sea razonable pedirle que lo aplique en tareas que pueden resolverse sin recurrir al teorema. No obstante, la regla de que el estudiante resuelva la tarea aplicando el teorema, no es de las que evita dramas e incluso puede resultar contraproducente para el aprendizaje matemático.

En el artículo donde se discute la actividad matemática en relación con el juego en un aula de infantil en Malta, el concepto sociocultural se utiliza desde la distinción vygotskiana entre lengua o cultura científicas y lengua o cultura cotidianas. Aquí, las situaciones didácticamente diseñadas de juego en clase permiten generar una cultura híbrida donde tanto lo científico como lo cotidiano tienen valor de instrumentos mediadores en el aprendizaje matemático del alumno. La flexibilidad de significados para el término sociocultural en la investigación en educación matemática se pone de relieve al contrastar las palabras y conceptos clave de este artículo con las palabras y conceptos clave de otros artículos del monográfico. Mientras que unos trabajos se centran en el análisis de aspectos antropológicos, sociológicos y filosóficos de cultura y de estructura social, otros trabajos se centran en el análisis de aspectos psicológicos de la acción y del desarrollo humano para relatar aprendizajes de matemáticas y procesos de enseñanza. En estos últimos, la estructura social es la de la interacción y la cultura es la del contexto inmediato donde se da la interacción, con sus artefactos o instrumentos mediadores materiales (e.g. las bolas del collar, la tarea) e inmateriales (e.g. la voz de la maestra, la tradición escolar). El artículo que indaga con futuras maestras acerca de la lengua matemática para enseñar la relación entre área y volumen considera desarrollos individuales de las participantes en el contexto sociocultural dado por la interacción con las tareas matemáticas del cuestionario y con una cultura escolar donde los significados para la noción de área en las primeras edades se limitan al reino de la geometría plana.

Nuestra memoria colectiva como miembros de la comunidad científica de investigación en educación matemática muestra que, históricamente, los énfasis en lo social (e.g. la interacción entre alumnos y con el profesor) y en lo cultural (e.g. los artefactos que configuran el contexto de la interacción) se han desplegado por separado de los énfasis en lo individual (e.g. el pensamiento del alumno, las creencias de la profesora). Esta introducción al monográfico es un buen espacio para reflexionar sobre los beneficios de no prescindir de los conocimientos producidos en estos distintos niveles. Son significativos los estudios que, desde las teorías cognitivas del aprendizaje humano, integran la influencia del contexto cultural y de la interacción social en el desarrollo de sistemas semióticos de interpretación de situaciones de enseñanza y de aprendizaje de matemáticas. Estos estudios prueban que el dominio de la investigación 
sociocultural en educación matemática también se está construyendo, como era de esperar, en la intersección con otros dominios del área. Algunos artículos del actual monográfico, por ejemplo, adoptan elementos de la cognición situada y rastrean la comunicación a través del cuerpo en los análisis de transcripciones como base de acceso a la mente, al pensamiento y a creencias de los participantes. No hay mención explícita a la dimensión corpórea de los significados, pero esta dimensión sí parece tenerse en cuenta a la luz de las interpretaciones de ciertos turnos de habla donde lo verbal literal y lo no verbal se selecciona, con puntos seguidos para omisiones (...), y se añade, con corchetes para añadidos [ ]. Las secuencias de acciones verbales y no verbales en la interacción, con transcripciones recreadas por el investigador, hacen que los límites entre lo individual (el cuerpo), y lo cultural y social (el mundo) sean bastante difusos.

Esta referencia a la interpretación de datos de transcripción apunta al tema de los métodos de análisis y a la pregunta de si hay métodos que puedan llamarse y reconocerse como socioculturales. Esta pregunta no es baladí. Fácilmente podríamos ponernos de acuerdo en que los análisis psicométricos se alejan del concepto sociocultural mientras que la etnografía de campo se acerca. Costaría más seguramente ponernos de acuerdo acerca de otros métodos. Los métodos cualitativos de análisis del contenido por ejemplo son epistemológicamente diversos; pueden aplicarse guiados por el concepto sociocultural según se tomen o no contenidos -unidades de análisis- relativos a especificidades de contextos de cultura y de interacción social. El análisis del contenido aplicado a documentos curriculares puede examinar la presencia de ciertos tópicos matemáticos de manera situada con respecto a la ubicación histórica y espacial de esos documentos (e.g. la geometría lineal de las carreteras rectas en la matemática escolar costarricense contemporánea). Para que hablemos de métodos socioculturales, debemos prestar atención a qué se prioriza en el proceso de comprensión. Los datos pueden ser productos directos o encapsulados de la cultura (e.g. libros de texto vs. respuestas escritas o habladas de un alumno a una tarea del libro, o de un futuro profesor al ítem de un cuestionario-entrevista), pero si no se recurre a la especificidad del contexto en el proceso de comprensión de esos datos (e.g. si el texto del libro no se pone en con-texto), es poco probable que el concepto sociocultural guíe el análisis. Distintos métodos de análisis pueden ser considerados socioculturales si en el proceso de comprensión de datos, es sustancial su ubicación en contextos de cultura y de estructura social. Los datos transcritos de una entrevista clínica a una futura profesora de matemáticas en su último curso de grado pueden ser analizados teniendo en cuenta la lengua de la estudiante como representación de su pensamiento, y como representación de las culturas de pedagogía matemática en las que ha participado a lo largo de las asignaturas de educación matemática del currículo universitario. Estas culturas devienen entonces datos.

Siguiendo aún con reflexiones sobre la determinación de métodos socioculturales, el artículo con datos de Arizona y de Nueva Zelanda es otra muestra de procesos de comprensión de transcripciones que se fundamentan en la ubicación de datos en contexto. Las transcripciones de interacciones en los talleres matemáticos de Arizona y en las clases de la escuela en Nueva Zelanda ilustran conocimientos matemáticos de jóvenes, madres, alumnos..., a la vez que estos conocimientos se explican en el contexto de cómo funcionan las comunidades de práctica, proporcionando a sus participantes conocimientos que pueden ser distintos a los regulados en los sistemas educativos oficiales. Aquí, para la comprensión de datos, los métodos de análisis del discurso en las transcripciones y en las observaciones de las investigadoras toman el contexto local físico y concreto en el tiempo- del taller y de la sesión de clase en los cuales se participa, además del contexto históricamente más amplio de la cultura, de la estructura social y 
de las reglas en las comunidades de los participantes. El grupo de métodos denominados de análisis del discurso pueden incluir, por tanto, métodos socioculturales según cuál sea la función que se la da al contex to de cultura y de estructura social en la comprensión de datos primarios y en la generación de nuevas fuentes de datos. A riesgo de insistir en algo trivial, vale la pena escribir una vez más que la atención al concepto sociocultural es la que hace que algunos métodos de análisis del contenido, de etnometodología, de etnografía, de análisis del contenido, de teoría fundamentada, de semiótica narrativa, de análisis del discurso, de combinaciones varias... sean socioculturales. Esto es así porque los métodos son siempre marco teórico y marco epistemológico.

Y puesto que una introducción no debería alargarse demasiado, acabo aquí. Confío que la lectura de los artículos del presente monográfico arroje muchos más aspectos de discusión, reflexión y crítica, o directamente de discusión reflexiva crítica. En cualquier momento de la historia de la investigación en educación matemática, conviene producir textos que nos ayuden a entender la construcción de dominios específicos y así a seguir construyéndolos. No es posible alcanzar una definición que englobe todas las apariciones del concepto sociocultural y sus aplicaciones en el área de investigación en educación matemática -ni por lo que sé en otras áreas como la de investigación en educación científica-, ni sería sensato plantear una definición simple que apenas explicara nada o que ahondara en la simplificación. Sin embargo, cuantas más apariciones y aplicaciones del concepto sociocultural estudiemos, más profunda será nuestra comprensión del concepto, del dominio y del área. Dada la variedad de significados 'puente', con suerte los artículos que siguen contribuirán a una mejor comprensión de la investigación sociocultural en educación matemática y de su papel complejo y diversificado en el área.

\section{Referencias}

Chevallard, Y. (1990). On mathematics education and culture: Critical afterthoughts. Educational Studies in Mathematics, 21, 3-27.

Glaser, B. G. (2001). The grounded theory perspective: Conceptualization contrasted with description. Mill Valley, CA: Sociology Press

Goodenough, H. W. (1971). Culture, language, and society. Reading, MA: AddisonWesley Modular Publications.

Lerman, S. (2000). The social turn in mathematics education research. En J. Boaler (Ed.), Multiple perspectives on mathematics teaching and learning (pp. 19-44). Westport, CT: Ablex Publishing.

Merleau-Ponty, M. (1964). Le visible et l'invisible. París: Gallimard.

Planas, N. (2010). Las teorías socioculturales en la investigación en educación matemática: Reflexiones y datos bibliométricos. En M. M. Moreno, A. Estrada, J. Carrillo, \& T. A. Sierra (Eds.), Actas del XIV Simposio de la Sociedad Española de Investigación en Educación Matemática (pp. 163-195). Lleida: SEIEM.

Radford, L. (2002). The seen, the spoken and the written. A semiotic approach to the problem of objectification of mathematical knowledge. For the Learning of Mathematics, 22(2), 14-23.

Skovsmose, O. (1994). Towards a philosophy of critical mathematics education. New York: Springer.

Vygotsky, L. S. (1978). Pensamiento y lenguaje. Madrid: Paidós. 